\title{
MATHEMATICAL MODELING OF THE HEAT TRANSFER PROCESS IN POROUS COOLING SYSTEMS UNDER INFLUENCE LOW ENERGY DISTURBANCES
}

\author{
Vyacheslav Ovchinnikov ${ }^{1, *}$, Eugenia Ruleva ${ }^{1}$, and Anatoliy Yakimov ${ }^{2}$ \\ ${ }^{1}$ National Research Tomsk State University, Scientific Institute of Applied Mathematics and \\ Mechanics, 634050 Tomsk, Russia \\ ${ }^{2}$ National Research Tomsk State University, Department of Physical and Computational Mechanics, \\ 634050 Tomsk, Russia
}

\begin{abstract}
The paper considers the interaction of a high-temperature flow with a transpiration cooling system exposed to low-energy perturbations of a coolant. It studies the possibility of controlling the heat exchange process in a composite material. The numerical calculations are compared with the experimental data.
\end{abstract}

\section{Introduction}

Vibration and oscillation processes accompany some natural phenomena and may be both harmful and beneficial. An oscillatory motion of gases and liquids intensifies a wide range of heat and mass transfer processes and stimulates the heat transfer of bodies, drying, diffusion, electrodeposition, etc. [1-3].

Transpiration cooling systems are widely used in engineering applications: evaporation systems, filters, thermal protection elements of flight vehicles [4-7]. The operation of such systems may be accompanied by some perturbations, such as acoustic oscillations, wall vibrations, pressure fluctuations and turbulent noises. Depending on the type of perturbations, oscillation amplitude and frequency, thermal and filtration parameters of porous materials may be distorted [7]. For example, in study [6], the thermal conductivity coefficient of a porous body increased in the presence of pressure fluctuations on its boundary.

The purpose of this paper is to carry out a theoretical study of transpiration cooling systems in the presence of low-energy perturbations and to compare the obtained findings with the known data.

\footnotetext{
*Corresponding author: ovc@niipmm.tsu.ru
} 


\section{Problem Statement}

Based on the approximate calculated relations obtained in [9], study [8] proposes a formula for finding a heat flow on the permeable plate in a turbulent gas flow:

$$
\frac{\mathrm{St}}{\mathrm{St}_{0}}=\frac{\gamma \exp \left(\frac{1-\operatorname{Pr}}{1+\operatorname{Pr}} \gamma\right)}{1+\frac{2 \gamma}{\operatorname{Pr}} \exp \left(\frac{\gamma}{1+\operatorname{Pr}}\right)-\exp \left(\frac{1-\operatorname{Pr}}{1+\operatorname{Pr}} \gamma\right)},
$$

where $\mathrm{St}=\frac{q_{w}}{(\rho v)_{e}\left(h_{e}-h_{w}\right)}, \quad \gamma=\frac{(\rho v)_{w}}{(\rho v)_{e} \mathrm{St}_{0}}, \quad \mathrm{St}_{0}=\frac{\left(\alpha / c_{p}\right)_{0}}{(\rho v)_{e}}, \operatorname{Pr}=\left(\frac{c_{p} \mu}{\lambda}\right)_{e}, \mathrm{St}$ is the Stanton number, Pr is the Prandtl number, $(\rho v)_{w}$ is the flow rate of the coolant gas in pores of the thermal protective material, $h$ is the enthalpy, $\lambda$ is the thermal conductivity coefficient, $c_{p}$ is the specific heat capacity, $\mu$ is the dynamic viscosity, $\alpha / c_{p}$ is the heat transfer coefficient. Superscripts and subscripts: $e$ is the outer edge of the boundary layer, $\mathrm{w}$ is the external heated surface of the body in flow, 0 means the absence of injection from the surface. Here, we consider the case when an injected substance and a substance of the primary flow are identical.

The varying (pulsating) convective heat flow acting on the thermal protective material for a definite time is assumed to occur in region 1 (fig. 1). Then, according to (1), we have:

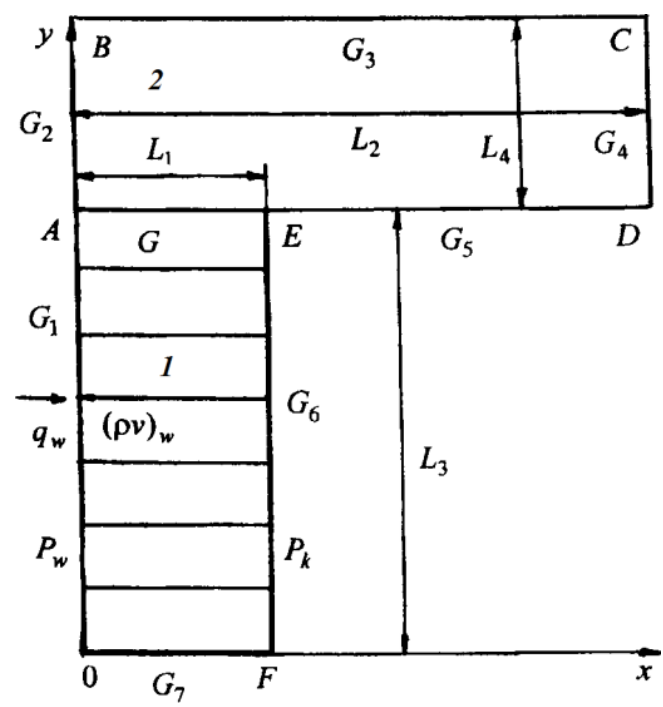

Fig. 1. Diagram of the flow around a body.

$$
q_{w}^{(1)}=\frac{(\rho v)_{w}\left(h_{e}-h_{w}^{(1)}\right) \exp \left(\frac{1-\operatorname{Pr}}{1+\operatorname{Pr}} b\right)}{1+\frac{2 b}{\operatorname{Pr}} \exp \left(\frac{b}{1+\operatorname{Pr}}\right)-\exp \left(\frac{1-\operatorname{Pr}}{1+\operatorname{Pr}} b\right)},
$$


where $b=\frac{(\rho v)_{w}}{\left(\alpha / c_{p}\right)_{0}},\left(\frac{\alpha}{c_{p}}\right)_{0}=\left(\frac{\alpha}{c_{p}}\right)_{\text {in }}\left[1+\frac{A \cos (f t)}{\left(\alpha / c_{p}\right)_{i n}}\right], f=2 \pi v, h_{w}^{(1)}=c_{p 2} T_{1 w}, A$ is the pulsation amplitude of the gas flow, $v$ is the frequency of periodic perturbations, $c_{p 2}$ is the specific heat capacity of the coolant gas at a constant pressure, $t$ is the time, $T$ is the temperature, $a_{1}, a_{2}$ are the constants. Superscripts and subscripts: in is the initial value (no pulsation), 1 and 2 below are, respectively, the frame and the gas in region 1 ; (1) and (2) above are, respectively, region 1 and 2 in fig. 1.

In curtain zone 2, we use the formula from [10] in the form of the Mugalev's modification, which takes into account the effect on injection on the heat flow [4]

$$
q_{w}^{(2)}=\left(\frac{\alpha}{c_{p}}\right)_{i n}\left(1-k_{1} b^{k_{2}}\right)\left(h_{e}-h_{w}^{(2)}\right), h_{w}^{(2)}=c_{p 2} T_{w}
$$

where $b=\frac{(\rho v)_{w}(y=0)}{\left(\alpha / c_{p}\right)_{i n}(\pi / 2-z)}, 1 \leq z \leq 1+L_{4} / L_{3}, k_{1}, k_{2}$ are the constants.

The thermal conductivity coefficient $\left(\lambda_{1}\right)$ for the frame of the thermal protective material is known from $[7,11]$

$$
\lambda^{\prime}=\frac{\pi c_{p 2} \rho_{2} \varphi B^{2} v}{\sqrt{2}}
$$

where $\rho_{2}$ is the density of the coolant gas, $\varphi$ is the porosity, $B$ is the pulsation amplitude for the frame wall of the thermal protective material.

The expression for effective viscosity $\mu_{e f}$ in the Darcy law (8) can be taken as Einstein's modification [12]:

$$
\mu_{e f}=\mu[1+C \cos (t f)]
$$

where $\mathrm{C}$ is the dimensionless coefficient $(0<C \leq 0.2)$.

The oscillation frequency $f$ can be found by the formula from [13]

$$
f=\frac{1}{B}\left(\frac{2 I}{\rho_{2} a}\right)^{0.5}
$$

where $I$ is the intensity of perturbations, $a$ is the speed of sound under normal conditions.

For the sake of simplicity, we make the following assumptions:

1) The Reynolds number in the incoming hypersonic air flow is sufficiently high $\left(\operatorname{Re}_{\infty} \gg 1\right)$ and the boundary layer has formed in the vicinity of the body surface;

2) The thermal protective material is a two-temperature medium, i.e. gas and condensed phases have different temperatures;

3) The mass flow rate at a normal to the plate in flow is significantly greater than the mass flow rate along this plate (fig. $1, L_{1}<<L_{3}$ );

4) A body interacting with a gas flow is not destroyed; no heterogeneous chemical reaction and phase transition occur inside or on its surface; 
5) The density of the gas phase is determined from the ideal gas equation;

6) The filtered gas is assumed to be uniform, with the molecular mass being close to that of the air mixture;

7) The contents of the injected gas and the gas in the incoming flow are identical.

8) The boundary problem in spatial variables is assumed to be two-dimensional in the case of heat propagation by conduction.

The flow rate of the coolant gas will be determined from the combined solution of the steady-state equation of continuity and the nonlinear Darcy law $[4,14]$. The fact is that high mass rates $(\rho v)_{w}(\operatorname{Re}>10)$ are associated with turbulent filtration behavior [14], which is characterized by the quadratic dependence of the pressure differential on the rate.

The problem of calculating heat and mass transfer parameters can be reduced to a system of conservation equations [7]:

in region 1 (see fig. 1)

$$
\begin{gathered}
\frac{\partial\left(\rho_{2} v \varphi\right)}{\partial x}=0, \\
\rho_{1} c_{p 1}(1-\varphi) \frac{\partial T_{1}}{\partial t}=\frac{\partial}{\partial x}\left[\lambda_{1}(1-\varphi) \frac{\partial T_{1}}{\partial x}\right]+\frac{\partial}{\partial y}\left[\lambda_{1}(1-\varphi) \frac{\partial T_{1}}{\partial y}\right]-A_{v}\left(T_{1}-T_{2}\right), \\
c_{p 2}\left(\rho_{2} \varphi \frac{\partial T_{2}}{\partial t}+\rho_{2} v \varphi \frac{\partial T_{2}}{\partial x}\right)=\frac{\partial}{\partial x}\left(\lambda_{2} \varphi \frac{\partial T_{2}}{\partial x}\right)+\frac{\partial}{\partial y}\left(\lambda_{2} \varphi \frac{\partial T_{2}}{\partial y}\right)+A_{v}\left(T_{1}-T_{2}\right), \\
\alpha_{n} \mu_{e f} v+\beta_{n} \rho_{2} \varphi v|v|=-\frac{\partial P}{\partial x}, \\
P=\frac{\rho_{2} R T_{2}}{M}, \lambda_{2}=\lambda_{2 i n} \sqrt{\frac{T_{2}}{T_{i n}}}, \mu=\mu_{i n} \sqrt{\frac{T_{2}}{T_{i n}}}
\end{gathered}
$$

in region 2 (solid steel edging in fig. 1)

$$
\rho c_{p} \frac{\partial T}{\partial t}=\frac{\partial}{\partial x}\left(\lambda \frac{\partial T}{\partial x}\right)+\frac{\partial}{\partial y}\left(\lambda \frac{\partial T}{\partial y}\right) .
$$

The system of equations (5)-(8), (10) must be solved by taking into account the following initial and boundary conditions:

$$
\left.T_{i}\right|_{t=0}=\left.T\right|_{t=0}=T_{i n}, i=1,2 .
$$

The following balance boundary conditions [15] are observed on the external heated surface of the porous plate $0 A$ (region 1 in fig. 1) [15].

$$
\begin{gathered}
\left(q_{w}^{(1)}-\varepsilon^{(1)} \sigma T_{1 w}^{4}\right)(1-\varphi)=-\left.\lambda_{1}(1-\varphi)\left(\frac{\partial T_{1}}{\partial x}\right)\right|_{G_{1}}, \\
q_{w}^{(1)} \varphi=-\left.\lambda_{2} \varphi\left(\frac{\partial T_{2}}{\partial x}\right)\right|_{G_{1}} ;
\end{gathered}
$$


on the symmetry axis $0 F$

$$
\left.\left(\frac{\partial T_{i}}{\partial y}\right)\right|_{G_{7}}=0, \quad i=1,2
$$

The thermal insulation conditions are specified on the surface of the solid steel shell $B C D$ :

$$
\left.\left(\frac{\partial T}{\partial y}\right)\right|_{G_{3}}=0,\left.\left(\frac{\partial T}{\partial x}\right)\right|_{G_{4}}=0
$$

on the internal surface $D E F[15]$ :

$$
\begin{gathered}
\left.\left(-\lambda \frac{\partial T}{\partial y}\right)\right|_{G_{5}}=\delta\left(\left.T\right|_{G_{5}}-T_{i n}\right),\left.\left(-\lambda_{1}(1-\varphi) \frac{\partial T_{1}}{\partial x}\right)\right|_{G_{6}}=\delta\left(\left.T_{1}\right|_{G_{6}}-T_{i n}\right), \\
\left.T_{2}\right|_{G_{6}}=\frac{\delta}{c_{p 2}(\rho v)_{w}}\left(\left.T_{1}\right|_{G_{6}}-T_{i n}\right)+T_{i n} .
\end{gathered}
$$

The ideal contact condition is specified on conjugation line $A E$ of regions 1 and 2:

$$
\left.\lambda_{1}(1-\varphi)\left(\frac{\partial T_{1}}{\partial y}\right)\right|_{G_{-}}=\left.\lambda\left(\frac{\partial T}{\partial y}\right)\right|_{G_{+}},\left.T_{1}\right|_{G_{-}}=\left.T\right|_{G_{+}},\left.T_{1}\right|_{G}=\left.T_{2}\right|_{G}
$$

on the external heated surface of edging 2 :

$$
q_{w}^{(2)}-\varepsilon^{(2)} \sigma T_{w}^{4}=-\left.\lambda\left(\frac{\partial T}{\partial x}\right)\right|_{G_{2}} .
$$

Pressure in the pores and the environment is equal both on the external and internal surfaces of region 1 .

$$
\left.P_{w}\right|_{G_{1}}=P_{e},\left.P\right|_{G_{6}}=P_{L_{1}} .
$$

Hereinafter, $x$ and $y$ are the transversal and longitudinal spatial coordinates, $P$ is the pressure, $v$ is the gas filtration rate in region $1, \rho$ is the density, $\delta$ is the heat transfer coefficient on the internal surface of the plate, $A_{v}$ is the volumetric heat transfer coefficient between the gas and the frame, $R$ is the universal gas constant, $\alpha_{n}$ and $\beta_{n}$ are respectively the viscosity and inertial coefficients in Darcy's law, $\sigma$ is the Stefan-Boltzmann constant, $\varepsilon^{(i)}, i=1,2$ is the emissivity of the surface of the thermal protective material and the edging, respectively, $M$ is the molecular mass of air, $L_{i}, i=$ 1, 2, 3, 4 are respectively the thicknesses and lengths of shells 1 and 2 in fig. $1, \mathrm{Pe}$ is the Peclet number, $\mathrm{Nu}$ is the Nusselt number. Superscripts and subscripts: $\infty$ is the value of the incoming gas flow at infinity, $k$ is the end time of thermal exposure, the prime above 
means the pulsation of heat transfer parameters, ef is the effective value, $L_{1}$ is the inner side of the body in flow, $n$ is nonlinearity.

\section{Numerical Method and Input Data}

The pressure on the external heated surface of the permeable body was assumed to be known from the experiment as a function of the longitudinal coordinate $y$ [11]. The pressure on the inside "cold" surface of the plate $\left(L_{1}\right)$ was taken as:

$$
P_{L_{1}}=k P_{e 0}
$$

The constant $k$ was specified to ensure the necessary flow rate of the coolant (in particular, not to reach the melting temperature of the steel shell, i.e. $1600 \mathrm{~K}$ [4]) in the thermal exposure area from $t=0$ to $t=t_{k}$.

The quasi-stationary equation of continuity $\rho_{2} \varphi v=-(\rho v)_{w}$ (the negative sign is explained by the fact that the normal coordinate $x$ is directed deep into the body (see fig. 1 ), and the coolant flows in the opposite direction) can be integrated along with the first equation (9), the nonlinear Darcy law (8) and the boundary conditions (20) to find the gas flow rate and the pressure in region $1[7,15]$ :

$$
\begin{aligned}
& (\rho v)_{w}(y)=\frac{\left[2 \beta_{n}\left(P_{L_{1}}^{2}-P_{w}^{2}\right) \varphi M D_{L_{1}} / R+E_{L_{1}}^{2}\right]^{0.5}-E_{L_{1}}}{2 \beta_{n} D_{L_{1}}}, \\
& P(x, y)=\left\{P_{w}^{2}+2 R(\rho v)_{w}\left[\beta_{n}(\rho v)_{w} D+E\right] / M \varphi\right\}^{0.5},
\end{aligned}
$$

where $D(x, y)=\int_{0}^{x} T_{2}(s, y) d s, E(x, y)=\alpha_{n} \int_{0}^{x} \mu_{e f} T_{2}(s, y) d s$.

The volumetric heat transfer coefficient $A_{v}$ between the gas and the frame was determined from the formulas [16]

$$
\mathrm{Nu}_{\mathrm{v}}=v_{1} \mathrm{Pe}^{\mathrm{v}_{2}}, \quad 0.5<\mathrm{Pe}<80, \quad v_{2}=1-1.3
$$

where $\mathrm{Nu}_{\mathrm{v}}=A_{v} l^{2} / \lambda_{2}, \mathrm{Pe}=(\rho v)_{w} l c_{p 2} / \lambda_{2}, l=\beta_{n} / \alpha_{n}$.

The boundary problem (6), (7), (10)-(19) was solved numerically using the locally onedimensional split-plot method [17]. The implicit, absolutely stable, monotone difference scheme was used with the cumulative approximation error $\mathrm{O}\left(\tau+H_{x}^{2}+H_{y}^{2}\right)$, where $H_{x}$ is the spatial step along the coordinate $x, H_{y}$ is the spatial step along the coordinate $y, \tau$ is the time step. The numerical method was tested for the basic option.

With all initial parameters being equal, the calculation was made for different spatial steps $H_{x}=0.5 \cdot 10^{-4} \mathrm{~m}, \quad H_{y}=2.4 \cdot 10^{-4} \mathrm{~m}, \quad h_{x 1}=2 \cdot H_{x}, \quad h_{x 2}=H_{x}, \quad h_{x 3}=H_{x} / 2$, $h_{x 4}=H_{x} / 4, h_{y 1}=2 \cdot H_{y}, h_{y 2}=H_{y}, h_{y 3}=H_{y} / 2, h_{y 4}=H_{y} / 4$. The temperature of the frame and the gas was recorded across the depth of the body at different times. In all cases, the problem was solved with the variable time step chosen on the assumption that the prescribed accuracy was equal for all spatial steps. The difference $\Delta=\max \left[\Delta_{T_{1}}, \Delta_{T_{2}}\right]$ in the 
relative temperature error was decreasing to reach $\Delta_{1}=10.3 \%, \Delta_{2}=5.4 \%, \Delta_{3}=2.6 \%$ at $t=t_{k}$. The calculation results presented below were obtained for spatial steps $h_{x 3}=H_{x} / 2, h_{y 3}=H_{y} / 2$.

The thermophysical and structural properties of the porous material were taken from for the sample made of sintered stainless steel powder [18, 19] and from [20] for air. The thermo-physical coefficients of solid steel (edging) $\lambda(T), c_{p}(T)$ as a function of temperature were taken from [21], while the values $\alpha_{n}$ and $\beta_{n}$ from $[11,18]$. The results presented below were obtained at $T_{\text {in }}=293 \mathrm{~K},\left(\alpha / c_{p}\right)_{\text {in }}=0.2 \mathrm{~kg} /\left(\mathrm{s} \cdot \mathrm{m}^{2}\right), A=0.03$ $\mathrm{kg} /\left(\mathrm{s} \cdot \mathrm{m}^{2}\right), \mu_{i n}=1.81 \cdot 10^{-5} \mathrm{~kg} /(\mathrm{m} \cdot \mathrm{s}), T_{e}=3600 \mathrm{~K}, \lambda_{e}=0.782 \mathrm{~W} /(\mathrm{m} \cdot \mathrm{K}), c_{p e}=4024$ $\mathrm{J} /(\mathrm{kg} \cdot \mathrm{K}), h_{e}=1.449 \cdot 10^{7} \mathrm{~J} / \mathrm{kg}, \rho_{e}=0.088 \mathrm{~kg} / \mathrm{m}^{3}, \mu_{e}=1.747 \cdot 10^{-4} \mathrm{~kg} /(\mathrm{m} \cdot \mathrm{s}), R=8.314$ $\mathrm{J} /($ mole $\cdot \mathrm{K}), B=10^{-3} \mathrm{~m}, M=29 \mathrm{~kg} / \mathrm{kmol}, \sigma=5.67 \cdot 10^{-8} \mathrm{~W} /\left(\mathrm{m}^{2} \cdot \mathrm{K}^{4}\right), L_{1}=2 \cdot 10^{-3} \mathrm{~m}$, $L_{2}=2 \cdot 10^{-2} \mathrm{~m}, L_{3}=9.5 \cdot 10^{-3} \mathrm{~m}, L_{4}=2 \cdot 10^{-3} \mathrm{~m}, \lambda_{1}=2.92+4.5 \cdot 10^{-3} \cdot T_{1} \mathrm{~W} /(\mathrm{m} \cdot \mathrm{K})$, $\rho_{1} c_{p 1}=\left(1252+0.544 \cdot T_{1}\right) \cdot 10^{3} \mathrm{~J} /\left(\mathrm{K} \cdot \mathrm{m}^{3}\right), \delta=100 \mathrm{~W} /\left(\mathrm{K} \cdot \mathrm{m}^{2}\right), \lambda_{2 i n}=0.0257 \mathrm{~W} /(\mathrm{m} \cdot \mathrm{K}), \rho$ $=7800 \mathrm{~kg} / \mathrm{m}^{3}, I=0-0.5 \mathrm{~kg} / \mathrm{s}^{3}, t_{k}=10 \mathrm{~s}, a=340 \mathrm{~m} / \mathrm{s}, \varphi=0.36-0.67, a_{1}=965.5, a_{2}=$ $0.0735, \varepsilon^{(1)}=0.85, \varepsilon^{(2)}=0.9, C=0.2, v_{1}=0.015, v_{2}=1$.

\section{Discussion of Numerical Solution Results}

Table 1 shows the experimental $T_{w}(0)$ [11] and calculated $T_{1 w}(0)$ temperatures of the external surface of permeable steel as a function of the coolant flow rate, parameter $k$ in the formula (21) with the initial data from Section 2 and the porosity $\varphi=0.36$, without periodic perturbations of the coolant gas $(I=0)$. As shown in Table 1, the difference in relative errors $(\Delta)$ between the calculated and experimental temperatures does not exceed $11.9 \%$.

Table 1. Relative error of the surface temperature as a function of the coolant flow rate and parameter $k$.

\begin{tabular}{|c|c|c|c|c|}
\hline $\boldsymbol{k}$ & $\begin{array}{c}(\rho v)_{w}(0), \\
\mathrm{kg} /\left(\mathrm{s} \cdot \mathrm{m}^{2}\right)\end{array}$ & $T_{w}(0), \mathrm{K}$ & $T_{1 w}(0), \mathrm{K}$ & $\Delta, \%$ \\
\hline 1.072 & 0.2 & 1224 & 1370 & 11.9 \\
\hline 1.092 & 0.4 & 1044 & 968 & 7.2 \\
\hline 1.105 & 0.6 & 792 & 763 & 3.7 \\
\hline 1.122 & 0.8 & 648 & 589 & 9.1 \\
\hline
\end{tabular}

Fig. 2 and 3 present the external temperature of the frame surface $T_{1 w}$ and the coolant flow rate $(\rho v)_{w}$ as a function of the longitudinal coordinate $y$ at $\varphi=0.67$ at the end time of thermal exposure $t=t_{k}$. Lines $1-4$ in fig. 2 and 3 correspond to the intensity of pulsations: $I=0,0.1,0.3,0.5$, i.e. for cases without or with pulsations for heat and mass transfer parameters in the formulas (2)-(4).

Analysis of lines 1-4 (fig. 2 and 3) shows that periodic perturbations taken into account may both increase or weaken the intensity of heat and mass transfer. This suggests that transpiration cooling systems are sensitive to pulsations of the coolant gas flow and that the heat transfer process in the thermal protective material can be controlled. 
Table 2 presents the temperature of the external surface of the porous steel frame $T_{1 w}$ as a function of the coolant flow rate at $\varphi=0.4, v=0$ and $v=5.2 \mathrm{~s}^{-1}$. The values $T_{1 w}(0)$ at $x=y=0$ correspond to the calculation results $T_{w}(0)$, while the values taken from [22] to the experimental results. The difference in the relative error between the numerical solution and the experiment does not exceed $18.2 \%$ at $v=0$ and $17.1 \%$ at $v=5.2 \mathrm{~s}^{-1}$.

Table 2. Relative error of the calculated temperature of the external frame surface as a function of the flow rate of the coolant gas without and with pulsations.

\begin{tabular}{|c|l|l|l|l|l|l|l|l|}
\hline $\mathrm{v}, \mathrm{s}^{-1}$ & \multicolumn{9}{|c|}{0} & \multicolumn{3}{c|}{5.2} \\
\hline $\begin{array}{c}(\rho v)_{w}(0), \\
\mathrm{kg} /\left(\mathrm{s} \cdot \mathrm{m}^{2}\right)\end{array}$ & 0.4 & 0.8 & 1.2 & 1.4 & 0.4 & 0.8 & 1.2 & 1.4 \\
\hline$T_{w}(0), \mathrm{K}$ & 1080 & 666 & 504 & 450 & 900 & 594 & 450 & 360 \\
\hline$T_{1 w}(0), \mathrm{K}$ & 933 & 578 & 412 & 373 & 752 & 492 & 375 & 339 \\
\hline$\Delta, \%$ & 13.6 & 13.2 & 18.2 & 17.1 & 16.4 & 17.1 & 16.6 & 5.8 \\
\hline
\end{tabular}

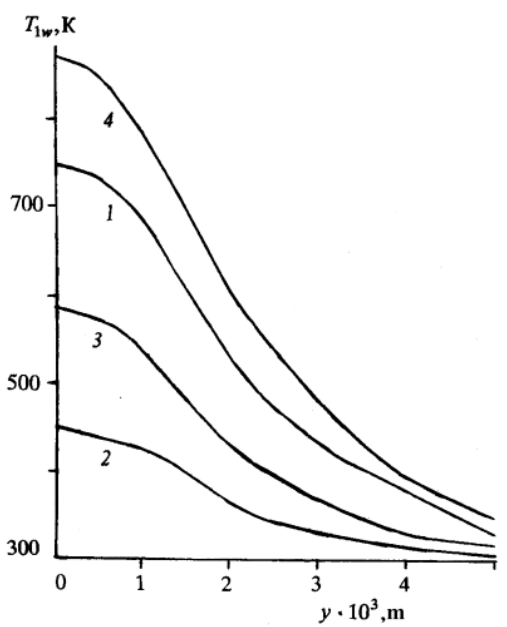

Fig. 2. The temperature of the external frame surface as a function of the longitudinal coordinate $y$ at time $t=t_{k}: 1-I=0,2-0.1,3-0.3,4-0.5$.

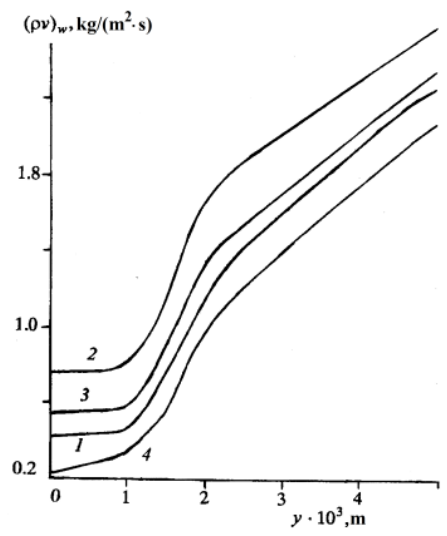


Fig. 3. Flow rate of the coolant gas as a function of the longitudinal coordinate $y$ at time $t=t_{k}$. The symbols are the same as in fig. 2.

On fig. 4 shows the temperature distribution of the porous steel frame $T_{1}$ (solid-curves) and gas $T_{2}$ (dashed curves) in depth $y$ layer at $x=0$ and $\varphi=0.4$ at different moments of time. Fig. 4 shows that for the balance of the boundary conditions (12) and (13) [15], have meaning for conservation laws, $T_{2 w}>T_{1 w}$. However, as we move deeper into the material quantity $T_{1}$ is greater $T_{2}$. It related by the sharp increase in the temperature of air at the surface and in a certain neighborhood because of the heat exchange with the external environment and the framework and greater thermal conductivity of the air than the skeleton of the porous steel. Then, as the heat transfer process of establishing the condensed phase is warming up faster, since the thermal conductivity of permeable steel two orders of magnitude higher than the thermal conductivity of the gas.

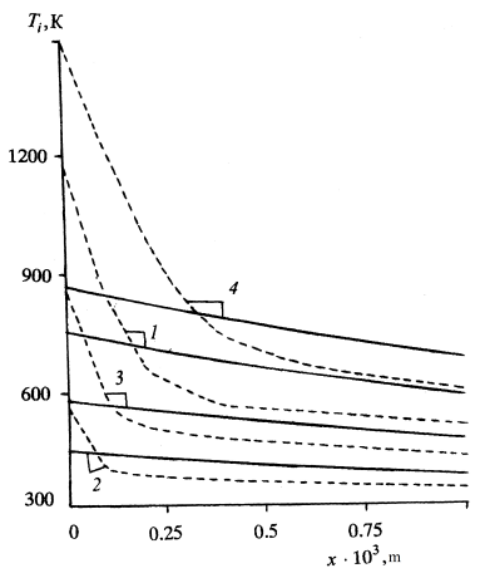

Fig. 4. Temperature distribution carcass gas layer depth with $x(y=0)$ at time $t=t_{k}$. The symbols are the same as in fig. 2.

\section{Conclusion}

The mathematical model was developed to calculate heat and mass transfer parameters in transpiration cooling systems with periodic perturbations of the coolant gas. Pulsations of the coolant gas flow have an ambiguous effect on the intensity of the heat mass transfer in transpiration cooling systems. The calculation results were proved to be consistent with the experimental data $[11,22]$ both in quantitative and qualitative terms.

\section{Acknowledgments}

The study was sponsored by the Ministry of Education and Science, Russian Federation, state assignment No. 9.1024.2014/k.

\section{References}

1. Yu.S. Kachanov, V.V. Kozlov, V.Ya. Levchenko, Origin of the Turbulence in the Boundary Layer (Novosibirsk, Science, 1982) 
2. V.E. Nakoryakov, A.P. Burdukov, A.M. Boldarev, P.N. Terleev, Heat and Mass Transfer in a Sound Field (Siberian Branch of the USSR Academy of Sciences, Novosibirsk, 1970)

3. O.V. Abramov, Crystallization of Metals in an Ultrasonic Field (Science, Moscow, 1972)

4. Yu.V. Polezhaev, F.B. Yurevich, Thermal Protection (Energy, Moscow, 1976).

5. V.M. Polyaev, V.A. Mayorov, L.A. Vasilyev, Hydrodynamics and Heat Transfer in Porous Elements of Flight Vehicle Structures (Mechanical Engineering, Moscow, 1988)

6. N. L. Azhishchev, V.I. Bykov, Proceedings of the Siberian Branch of the USSR Academy of Sciences. Engineering Science Series 21, 27 (1987)

7. A.M. Grishin, A.N. Golovanov, V.I. Zinchenko, E.K. Efimov, A.S. Yakimov, Mathematical and Physical Modeling of Thermal Protection (Tomsk University Publishing House, Tomsk, 2011)

8. V.D. Sovershenny, J. Eng. Phys. 12, 538 (1967)

9. V.D. Sovershenny, Izv. Akad. Nauk SSSR. Mech. Fluids Gases 3, 45 (1966)

10. A.V. Bureev, V.I. Zinchenko, Applied Mech. and Eng. Phys. 6, 72 (1991)

11. A.N. Golovanov, E.V. Ruleva, A.S. Yakimov, High-Temperature Thermophys. 49, 914 (2011)

12. A. Einstein, M. Smoluchowski, Brownian Motion (Main Publishing House of General Engineering Literature, Moscow-Leningrad, 1936)

13. A.N. Golovanov, Physics of Combustion and Explosion 35, 67 (1999)

14. R.A. Andrievsky, Porous Metal-Ceramic Materials (Metallurgy, Moscow, 1964)

15. A.M. Grishin, A.N. Golovanov, A.S. Yakimov, Appl. Mech. Eng. Phys. 4, 141 (1991)

16. V.F. Zanemonets, V.I. Rodionov, Heat and Mass Transfer. Minsk International Forum, 7, 42 (1988)

17. A.A. Samarsky, Introduction to the Theory of Difference Schemes (Science, Moscow, 1971)

18. A.N. Golovanov, J. Eng. Phys. 66, 695 (1994)

19. O.M. Alifanov, A.P. Tryanin, A.L. Lozhkin, J. Eng. Phys. 52, 461 (1987)

20. N.B. Vargaftik, Guide to Thermophysical Properties of Gases and Fluids (Physical and Mathematical State Edition, Moscow, 1963)

21. V.F. Zinovyev, V.I. Rodionov, Thermophysical Properties of Metals at High Temperatures. Handbook (Metallurgy, Moscow, 1989)

22. A.N. Golovanov, E.V. Ruleva, Herald of the Tomsk State University. Mathematics and Mechanics 14, 85 (2011) 